\title{
Center-Shift Method for the Characterization of Dielectric Charging in RF MEMS Capacitive Switches
}

\author{
Rodolf W. Herfst, Peter G. Steeneken, H. G. A. (Bert) Huizing, and Jurriaan Schmitz, Senior Member, IEEE
}

\begin{abstract}
Radio frequency (RF) micro-electro-mechanical systems (MEMS) capacitive switches show great promise for use in wireless communication devices such as mobile phones, but for the successful application of these switches their reliability needs to be demonstrated. One of the main factors that limits the reliability is charge injection in the dielectric layer (SiN) which can cause irreversible stiction of the moving part of the switch.

We present a way to characterize charge injection. By stressing the dielectric with electric fields on the order of $1 \mathrm{MV} / \mathrm{cm}$, we inject charge in the dielectric and measure the effects it has on the $C V$ curve. Instead of conventionally measuring the change of the pull-in voltage, the presented center shift method measures the change of the voltage at which the capacitance is minimal. This way, the measurement method does not influence the charge injected by the stress voltage. Another advantage is that the measurement of the amount of injected charge is not influenced by changes in the width of the $C V$ curve. These two advantages make it possible to test RF-MEMS capacitive switches in a more accurate way.
\end{abstract}

Index Terms-Capacitive switch, charging, dielectric, radio (RF) micro-electro-mechanical systems (MEMS), reliability.

\section{INTRODUCTION}

$\mathbf{R}$ ADIO frequency (RF) micro-electro-mechanical systems (MEMS) capacitive switches show great potential for use in wireless communication devices such as mobile phones. This is due to the low loss, high linearity, good power handling, and low power consumption of the switches [1]. Fig. 1 shows a schematic representation of an RF MEMS capacitive switch. The top electrode is suspended by (tiny) springs and can be pulled down by applying a voltage across the air gap between the two electrodes. Above a certain voltage, the balance between the attracting electrostatic force $F_{E}$ and restoring spring force $F_{\text {spring }}$ becomes unstable and the switch closes, which is marked by a sudden increase in the capacitance of the switch (Fig. 2). This voltage is called the pull-in voltage $V_{\mathrm{pi}}$. A dielectric layer prevents dc current flow. Once closed, the electric forces are much higher due to the shorter distance between the electrodes, and the switch will only open again if the voltage is lowered below the so-called pull-out voltage $\left(V_{\mathrm{po}}\right) . V_{\mathrm{pi}}$ and $V_{\mathrm{po}}$ can be found by measuring the hysteresis present in the capacitance-voltage curve of the switch (Fig. 2). Since the electrostatic force is proportional to the voltage squared, pull-in and pull-out

Manuscript received February 28, 2007; revised August 2, 2007.

R. W. Herfst, P. G. Steeneken, and H. G. A. Huizing are with NXP Semiconductors Eindhoven, 5656 AA Eindhoven, The Netherlands (e-mail: rudolf. herfst@nxp.com; peter.steeneken@NXP.com; bert.huizing@nxp.com).

J. Schmitz is with MESA+ Research Institute, University of Twente, $7500 \mathrm{AE}$

Enschede, The Netherlands (e-mail: J.Schmitz@utwente.nl).

Digital Object Identifier 10.1109/TSM.2008.2000285

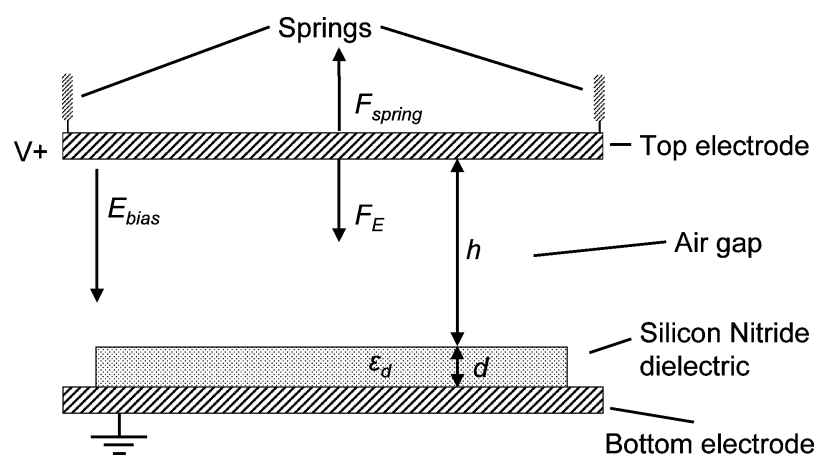

Fig. 1. Schematic representation of RF MEMS. Top electrode of a parallel plate capacitor can be pulled down by applying a voltage greater than the pull-in voltage $\left(|V|>\left|V_{\mathrm{pi}}\right|\right)$, which is pulled up again by springs if voltage is lowered beneath the pull-out voltage $\left(|V|<\left|V_{\mathrm{po}}\right|\right)$.

occur for both positive and negative applied voltage, indicated in the figure by $V_{\mathrm{pi}}^{+}, V_{\mathrm{po}}^{+}, V_{\mathrm{pi}}^{-}$, and $V_{\mathrm{po}}^{-}$.

In the closed state, the electric field in the dielectric layer is on the order of $1 \mathrm{MV} / \mathrm{cm}$. Because of this high field, charge is injected into the dielectric, which changes both the electric field present in the gap between the two plates and the amount of charge in the bottom and top electrode, thereby influencing the electrostatic force (Fig. 3). In case of a positive trapped charge, negative image charges will appear on the top and bottom electrode, which reduces the total amount of charge on the electrodes if a positive voltage is applied. The net effect of injected positive charges is a positive shift of the $C V$ curve [2]-[4], which in turn affects the pull-in and pull-out voltages: $V_{\mathrm{pi}} \Rightarrow$ $V_{\text {pi }}+V_{\text {shift }}$ and $V_{\text {po }} \Rightarrow V_{\text {po }}+V_{\text {shift }}$ (Fig. 4). Here, $V_{\text {shift }}$ is linearly proportional to the amount of injected charge. A large amount of injected charge can even lead to failure of the switch due to stiction of the top electrode to the dielectric. This happens when $V_{\mathrm{po}}^{-}$becomes positive or when $V_{\mathrm{po}}^{+}$becomes negative. In that case, the switch can be in the closed branch of the hysteresis curve even at $0 \mathrm{~V}$. For instance, if $V_{\mathrm{po}}^{-}>0$, and the switch is closed by applying a voltage above $V_{\mathrm{pi}}^{+}$, it will not open again when the voltage is suddenly set to zero.

In this paper, we consider three different methods for measuring the shift of the $C V$ curve with a low-frequency measurement setup. Two are based on determining $V_{\mathrm{pi}}$ and one is based on determining the center shift of the $C V$ curve, which, to our knowledge, has not been proposed before. The influence that the measurements have on the switches is investigated and is compared with results obtained with a fast RF- $C V$ measurement setup. The new method, in contrast to other methods, does 


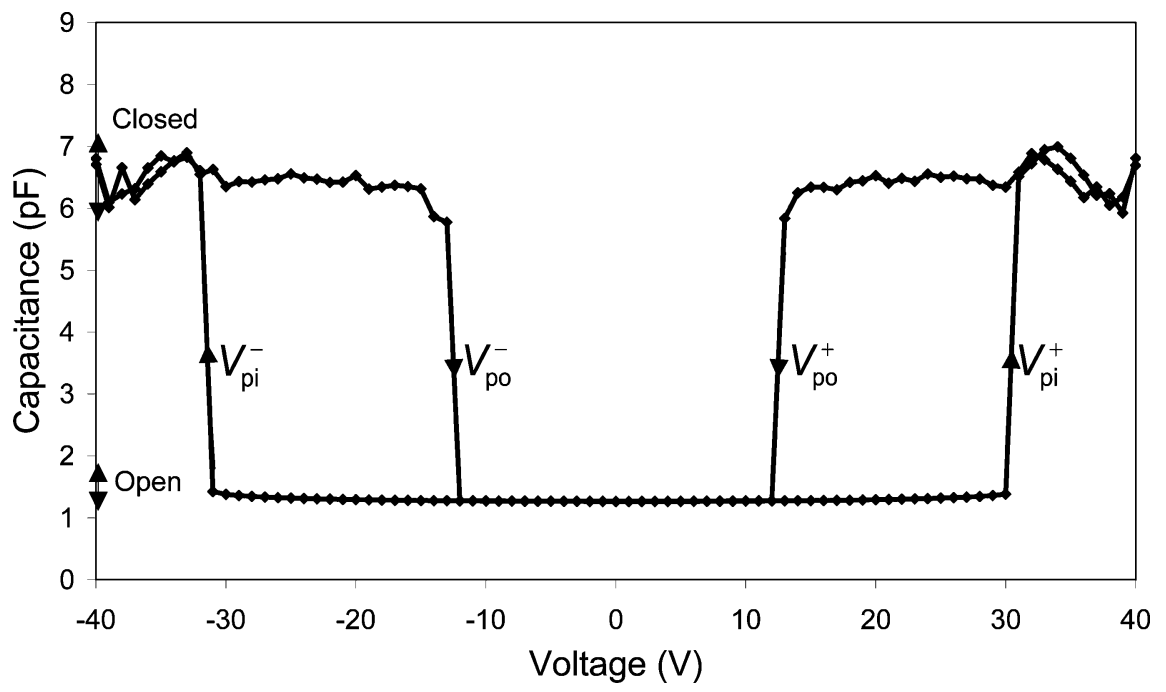

Fig. 2. Typical $C V$ curve of RF MEMS capacitive switch. By increasing voltage, top electrode is pulled down and capacitance increases. Above $|V|=\left|V_{\mathrm{pi}}\right|$ switch closes. When voltage is lowered again, switch opens for $|V|<\left|V_{\text {po }}\right|$.

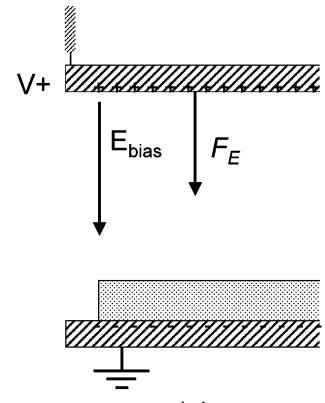

(a)

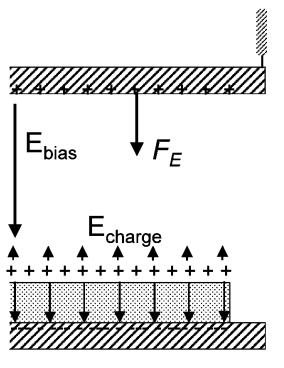

(b)
Fig. 3. E-field and electrostatic force in parallel plate capacitor with positive bias voltage. (a) No fixed charges in dielectric. (b) Fixed surface charge in and on dielectric.

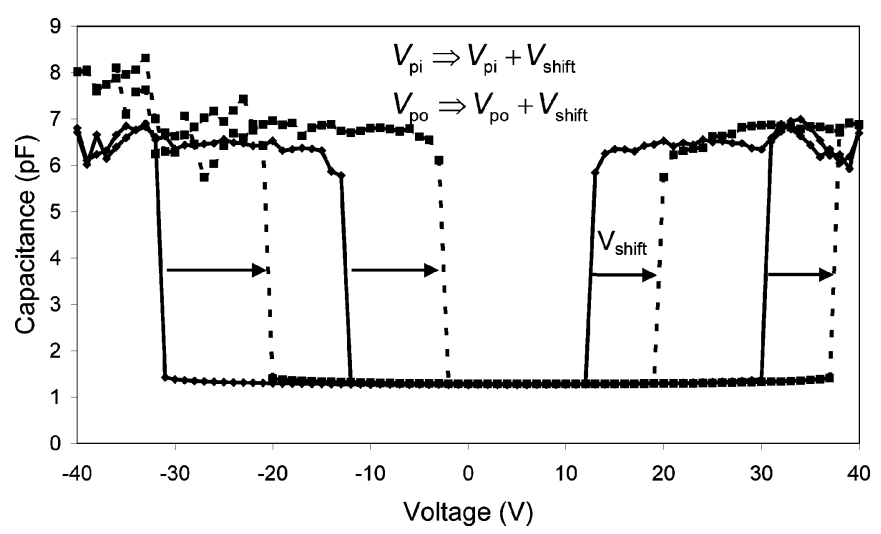

Fig. 4. $C V$ curve before (black) and after (grey) switch has been stressed at $65 \mathrm{~V}$ for $727 \mathrm{~s}$.

not degrade the switches. This makes it possible to test the impact that changes in the RF MEMS manufacturing process have on the reliability more accurately. The new method is used to characterize dielectric charging at several voltages.

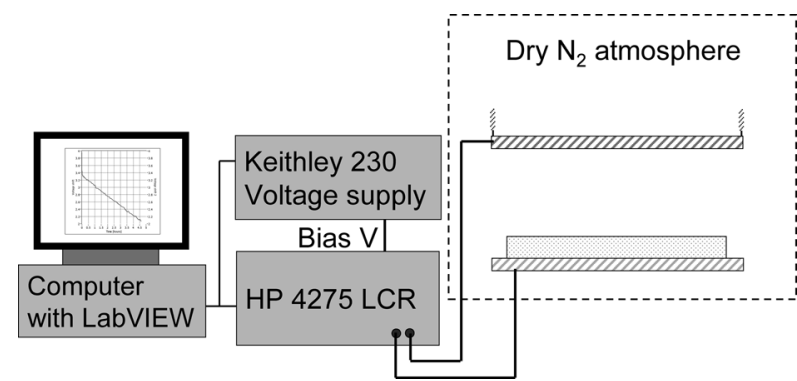

Fig. 5. Schematic view of measurement setup. LabVIEW computer program controls HP4275 LCR meter and Keithley 230 V Programmable Voltage Source. LCR meter is connected to RF MEMS capacitive switches, which are stressed and measured in a dry $\mathrm{N}_{2}$ atmosphere.

\section{MEASURING DiELECTRIC CHARGES}

\section{A. Setups and Measurement Methods}

To study charge injection, $V_{\text {shift }}$ is measured as a function of stress voltage and time. One of the setups with which these measurements can be done is depicted schematically in Fig. 5. To avoid moisture from influencing the measurements, the switches are stressed and measured in a dry nitrogen environment at atmospheric pressure. A bias voltage is provided by a Keithley 230 Programmable Voltage Source to a HP4275 LCR meter which is then used to measure the capacitance as function of voltage. The test signal of the LCR meter was $4 \mathrm{MHz}$. A picture of the switches which were used for the measurements is shown in Fig. 6.

The switches are stressed and $V_{\text {shift }}$ is measured periodically. To measure $V_{\text {shift }}$, three methods are considered.

1) Whole $C V$ curve method: Measure the $C V$ curve with equidistant steps from below $V_{\mathrm{pi}}^{-}$to above $V_{\mathrm{pi}}^{+}$back to below $-V_{\mathrm{pi}}$ again. Compare to the first $C V$ curve by determining the pull-in and pull-out voltages [5], [6]. $V_{\text {shift }}$ is determined by $V_{\text {shift }}=V_{\mathrm{pi}}-V_{\mathrm{pi}}^{t=0}$.

2) Successive approximation method: Similar to the whole $C V$ curve method, this method searches for the value of $V_{\text {pi }}$ by an algorithm based on successive approximation. 


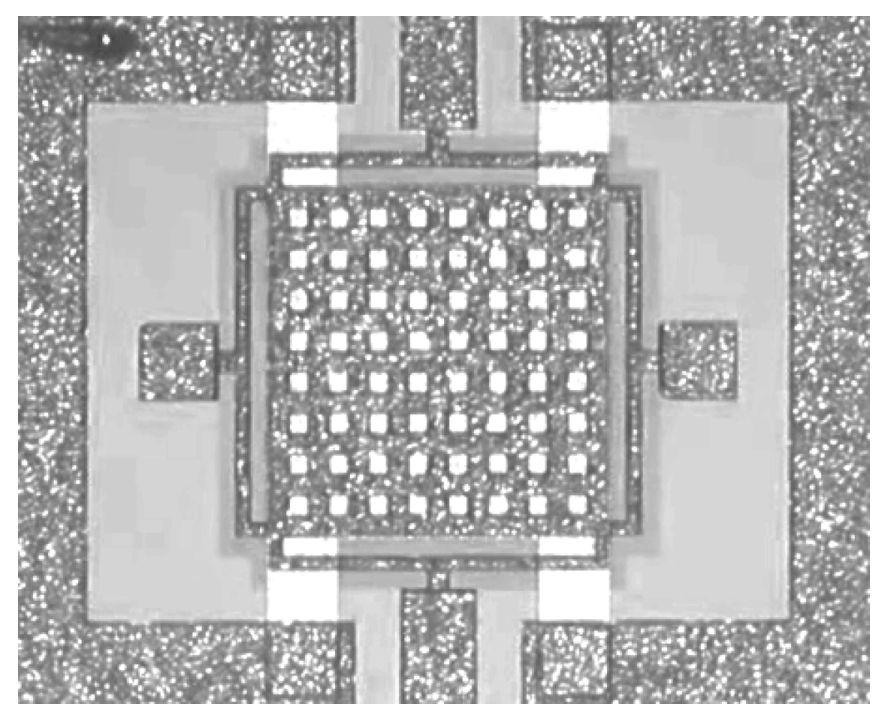

Fig. 6. One of the switches used in measurements. Top electrode of device under study is $0.46 \times 0.46 \mathrm{~mm}^{2}$, dielectric has thickness of approximately 0.4 $\mu \mathrm{m}$, and air gap is approximately $5.2 \mu \mathrm{m}$.

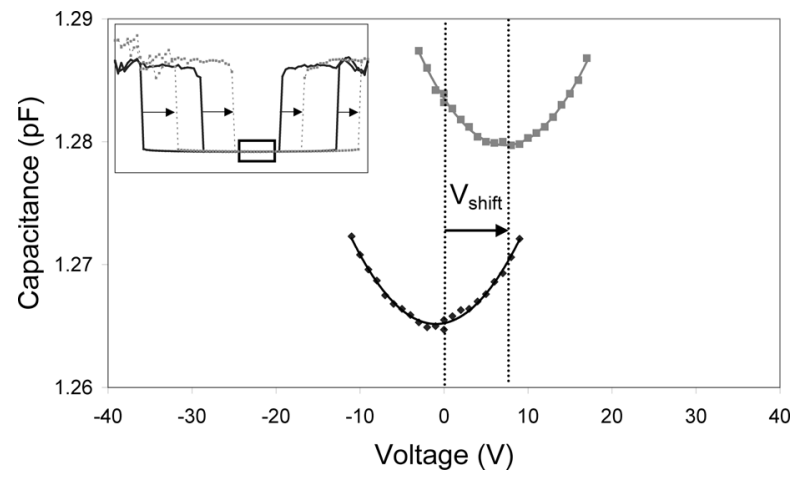

Fig. 7. Center of $C V$ curve before (black) and after (grey) switch has been stressed at $65 \mathrm{~V}$ for $727 \mathrm{~s}$. By fitting parabola through data, center $V_{\text {shift }}$ can be accurately determined.

Note that after each guess for the pull-in voltage the switch must be allowed to open again. Again, $V_{\text {shift }}$ is determined by $V_{\text {shift }}=V_{\mathrm{pi}}-V_{\mathrm{pi}}^{t=0}$.

3) Center shift method: Only measure the shift of the center (opened switch) part of the $C V$ curve, and calculate the voltage at which the capacitance has the lowest value. This is done by fitting a parabola $C(V)=a \cdot\left(V-V_{\text {shift }}\right)^{2}+$ $C_{\text {open }}$ through the center of the $C V$ curve (Fig. 7). Since the electrostatic force is proportional to $\left(V-V_{\text {shift }}\right)^{2}$ [7], $C(V)$ is symmetric around $V=V_{\text {shift }}$, therefore this fitted parabola accurately determines $V_{\text {shift }}$. To our knowledge, this is a new method. A known published noncontact method [8] differs from our method: it requires RF measurements and $V_{\text {shift }}$ is determined by manually tuning the bias voltage for minimum capacitance. This method is applicable for both low-frequency LCR meters and RF equipment and requires no manual tuning of the bias voltage.

The whole $C V$ curve method has the disadvantage that for each measurement of $V_{\text {shift }}$, the capacitance has to be measured at least $4 \times V_{\mathrm{pi}} / V_{\text {step }}$ times, where $V_{\text {step }}$ is the voltage step size with which the $C V$ curve is measured. Since the LCR meter

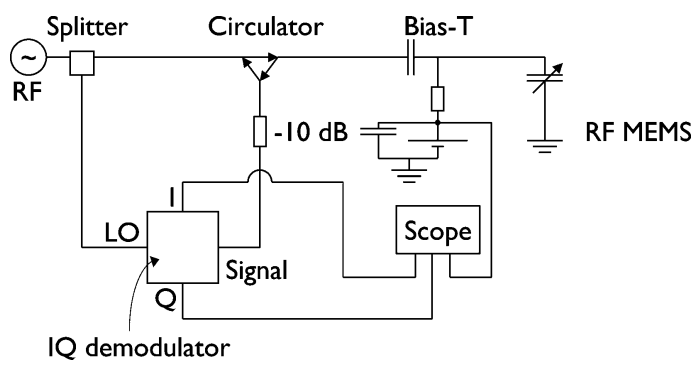

Fig. 8. Schematic of 1-port S-parameter measurement system. Phase and amplitude of reflected RF signal are measured with an IQ-demodulator, from which capacitance can be determined.

takes roughly $1 \mathrm{~s}$ to accurately measure the capacitance, measuring $V_{\text {shift }}$ takes a significant amount of time so that during the measurement of $V_{\text {shift }}$ charge can leak away again. Also, voltages above $V_{\text {pi }}$ have to be applied so that the switch "sees" additional stress during the determination of $V_{\text {shift }}$. The successive approximation method is faster and also more accurate, but still has one of the problems of the whole $C V$ curve method: during the measurements voltages above $V_{\mathrm{pi}}$ have to be applied.

By contrast, the center shift method is faster and has no risk of charging the dielectric further during the determination of $V_{\text {shift }}$. In our case, we measured the center part from the $C V$ curve in 17 steps, significantly less than the 162 steps required to measure a complete $C V$ curve from -40 to $+40 \mathrm{~V}$ with $1 \mathrm{~V}$ resolution and also less than the 26 steps used for the successive approximation method. Also, since a large $V_{\text {shift }}$ may prevent the switch from opening at $0 \mathrm{~V}$, the measurement voltage is first set to the previous value of $V_{\text {shift }}$, rather than $0 \mathrm{~V}$. Due to this step, the switch will be forced into the open branch of the hysteresis curve and open. The new value of $V_{\text {shift }}$ is determined by measuring $C(V)$ around the previous measured value of $V_{\text {shift }}$. This way, $V_{\text {shift }}$ can even be measured when $V_{\text {po }}^{L}>0$ (or $V_{\mathrm{po}}^{R}<0$ ), thereby extending the range of voltage shifts that can be measured.

To reduce the measurement time of $C V$ curves, a second setup with which we can do reliability measurements was constructed. Here, instead of an off-the-shelf LCR meter, a custom $\mathrm{RF}$ setup is used to measure the $C V$ curve. The bias voltage is provided by an amplified signal from a function generator. Compared to the LCR setup this RF setup has several advantages as well as disadvantages: while the capacitance can be measured very quickly, it is more difficult to implement, requires some tedious calibration steps, and requires the capacitive switches to have a layout which is compatible with RF-probes. It also has a lower signal-to-noise ratio.

With the RF setup, capacitances are measured with a discrete single-frequency 1-port S-parameter measurement system based on the description given by Nieminen et al. [9] which is schematically depicted in Fig. 8. An RF signal (890 MHz) from a signal generator is split in two. One part goes into the local oscillator of the IQ-demodulator. The other part of the signal passes through a circulator, after which a bias voltage is added to the signal. The signal then reflects back from the RF MEMS capacitive switch. The reflected signal passes through the bias-T and the circulator to the RF-in port of the IQ-demodulator. The amplitude and phase of the reflected signal are determined from the I and Q signals using an oscilloscope. A 10-dB 


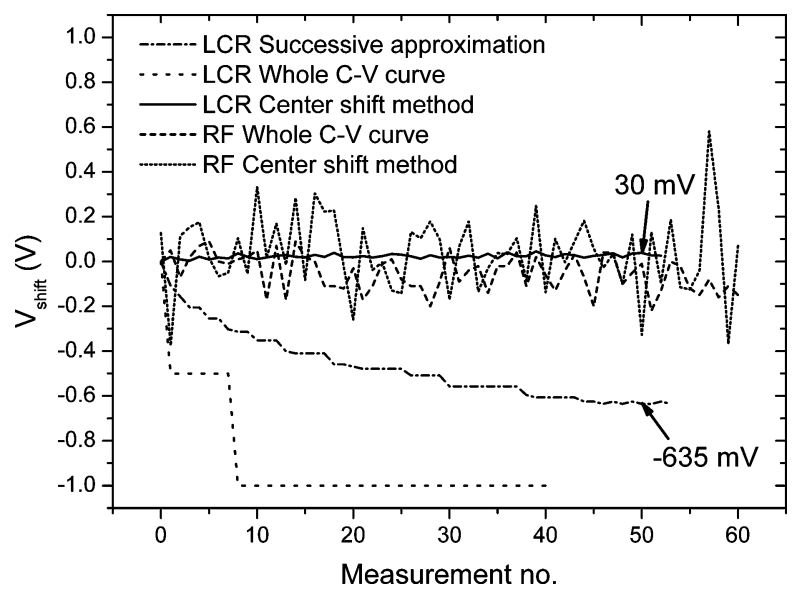

Fig. 9. Effect of measurements on voltage shift determined by measuring change in pull-in voltage and by determining center of $C V$ curve when applying no stress voltage. Before switch was stressed, an initial $V_{\text {shift }}$ of $-444 \mathrm{mV}$ measured. This was subtracted from center shift results for better comparison with two other methods.

attenuator (Fig. 8) between the circulator and the signal port of the IQ-demodulator reduces the effect of small nonlinearities in the IQ-demodulator. By first performing an open-short-load calibration, the capacitance can be calculated from the measured reflection. The capacitance measurement speed is high, and the $C V$ curve measurement time is limited by the mechanical response time of the MEMS: if the measurement is performed too quickly, the voltage changes while the switch is closing. In this case, the flanks at pull-in and pull-out in the $C V$ curve are not vertical. To eliminate this effect, we measure a whole $C V$ curve in $400 \mathrm{~ms}$ and parabolas for the center shift method in $100 \mathrm{~ms}$. To increase the accuracy of the measurement, the average of two $C V$ curves is taken for the whole $C V$ curve method. The parabolas for the extraction of the center shift are measured and averaged eight times. The successive approximation method is not used with the RF setup.

\section{B. Results: Comparison of Measurement Setups and Methods}

In this section, we compare the influence of the different measurement methods and setups on the measurement results. In Fig. 9, the effects and reproducibility of the determination of $V_{\text {shift }}$ with the different methods and setups are shown. No stress voltage was applied between consecutive measurements of $V_{\text {shift }}$. As can be seen, the measured values of $V_{\text {shift }}$ obtained with the center shift method on the LCR setup only show a negligible drift (approximately $30 \mathrm{mV}$ at the 50th measurement). If we apply a linear fit to this data we find a drift rate of $0.3 \mathrm{mV}$ per measurement and an offset of $16 \mathrm{mV}$. The measured values of $V_{\text {shift }}$ obtained with the whole $C V$ curve (also with the LCR setup) and successive approximation method show a significant change when measured repeatedly: with the successive approximation method was $-635 \mathrm{mV}$ after the 50th measurement, more than 21 times larger than the drift of the center shift method. The noise on $V_{\text {shift }}$ is also low with the center shift method: the standard deviation with respect to the linear fit is $8.2 \mathrm{mV}$.
With the RF setup, there is no significant drift for both the center shift method and for the whole $C V$ curve method. This shows that if you measure the $C V$ curve fast enough, the whole $C V$ curve method can also be used. However, due to the lower signal-to-noise ratio of the discrete RF setup and the noise on the amplified voltage from the signal generator, there is a larger spread in the measured voltage shift. The standard deviation on the values of $V_{\text {shift }}$ determined with the RF center shift method is $170 \mathrm{mV}$, which is mainly due to the uncertainty of the parabola fit. Although the accuracy of the parabola fit obtained with the RF setup is worse than the results obtained with the LCR setup ( $170 \mathrm{mV}$ for the RF setup versus $8.2 \mathrm{mV}$ for the LCR meter), the RF setup faster ( 2.5 versus $17 \mathrm{~s}$ ). The standard deviation in the values of $V_{\text {shift }}$ determined from the change in $V_{\mathrm{pi}}$ is $80 \mathrm{mV}$.

All in all, the results clearly show the necessity of measuring charging effects in the open state by using the center shift method or to very rapidly measure the $C V$ curve to avoid that the measurement itself influences the result by using a fast $\mathrm{RF}$ setup. They further show that the LCR setup is slower and more accurate than the RF setup.

In Fig. 10, we have used the center shift method to determine the shifts in the $C V$ curve due to three different stress voltages on 20 devices which were not used prior to the stress test. As one would expect, a higher stress voltage results in a faster and larger change of $V_{\text {shift }}$. According to Fig. 9, if these measurements had been done with the successive approximation method instead of the center shift method, a drift of about $-470 \mathrm{mV}$ would have been induced during the 20 measurements of $V_{\text {shift }}$ which were conducted during the $30 \mathrm{~min}$ of applied stress. This would give a significant deviation: at $50 \mathrm{~V}$ the voltage shift of the $C V$ curve after 27 min was between 1.0 and $1.8 \mathrm{~V}$. Even at $60 \mathrm{~V}$, the drift part would have been $7.5 \%$ of the measured value. With the center shift method a drift of less than $6 \mathrm{mV}$ is expected. This indicates that the use of the center shift method is much better suited to characterize charging, especially at lower stress voltages. We also observe a spread in the measurement data. It is speculated that this is due to small variations in the composition, thickness of the dielectric, and surface roughness of the top electrode, which leads to variations in the electric field and charging current.

In Fig. 11, the RF setup is used to measure $V_{\text {shift }}$ (determined with the center shift method) and the positive and negative pull-in voltage extracted from the whole $C V$ curve as function of stress time at a stress voltage of $45 \mathrm{~V}$. Closer inspection reveals that the curves get closer together after more voltage stress has been applied. To make this clearer, the voltage axis in Fig. 11 has been divided into three parts. Additionally, the $V_{\text {shift }}$ has also been determined as $\left(V_{\mathrm{pi}}^{+}+V_{\mathrm{pi}}^{-}\right) / 2$. As can be seen, the results almost overlap with the results from the fitted parabola.

The phenomena of $C V$ curve narrowing has been observed before and an explanation for this has been proposed by Rottenberg et al. [10]: if the injected charge is laterally inhomogeneous, the internal $E$-field caused by the charge can never be completely compensated by applying a laterally homogeneous $E$-field. The net result is that there are two effects: the center of 


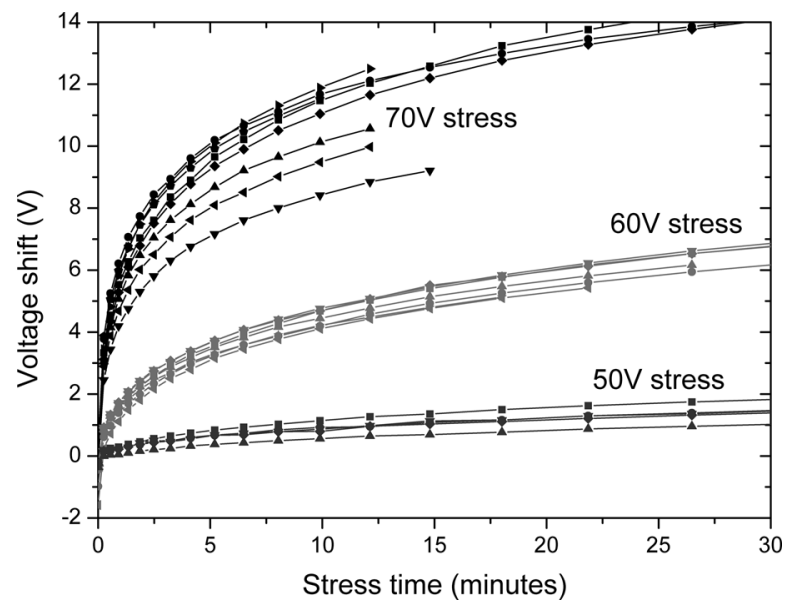

Fig. 10. Shift of $C V$ curve as function of time at room temperature for 50 , 60 , and $70 \mathrm{~V}$ stress for 20 devices, measured with center shift method on LCR setup. Each device was not used prior to stress test.

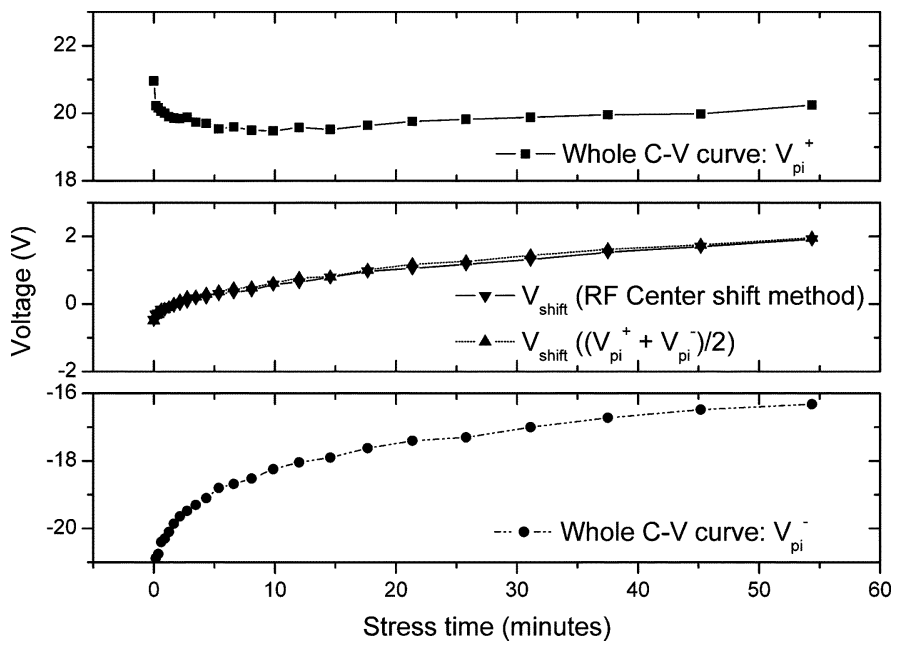

Fig. 11. $V_{\mathrm{pi}}^{-}, V_{\text {shift }},\left(V_{\mathrm{pi}}^{+}+V_{\mathrm{pi}}^{-}\right) / 2$, and $V_{\mathrm{pi}}^{+}$as function of stress time as measured with RF setup at stress voltage of $45 \mathrm{~V}$.

the $C V$ curve proportional undergoes a shift proportional to the mean of the injected charge, while the lateral inhomogeneity results in an extra contribution in the electrostatic force, which is proportional to the standard deviation of the amount of injected charge. This causes the $C V$ curve to narrow. Other causes for $C V$ curve narrowing could be changes in the spring constant and the gap height.

From the observed $C V$ curve narrowing we can conclude that even if the $C V$ curve can be measured fast enough so that virtually no charge is injected during the $C V$ measurement, $V_{\text {shift }}$ should not be determined as a change in one of the pull-in voltages, but from the shift of the center of the $C V$ curve. This center shift can be determined by fitting a parabola or from $\left(V_{\mathrm{pi}}^{+}+V_{\mathrm{pi}}^{-}\right) / 2$.

\section{CONCLUSION}

The effects of charge injection in the dielectric layer of an RF MEMS capacitive switch are studied using a center shift measurement method which is both accurate $(8.2 \mathrm{mV}$ standard deviation on the LCR setup) and has less influence on the device under test than the commonly used procedure of measuring the change in the pull-in voltage. By measuring only the central part of the $C V$ curve and fitting a parabola $C(V)=a \cdot(V-$ $\left.V_{\text {shift }}\right)^{2}+C_{\text {open }}$ to the data, $V_{\text {shift }}$ can be determined while the switch is in the open position, so that no additional charge is injected during the measurement, thereby separating the effect of intentionally induced stress and the stress effect that the measurement has on the device under test. This is especially important if dielectric charging at lower stress voltages is studied: at these lower voltages the $C V$ curve shifts are smaller and slower, thereby requiring more accurate measurements. Another advantage is that if the $C V$ curve narrows due to laterally inhomogeneous charging, a $V_{\text {shift }}$ determined with the center shift method will not be affected. This allows homogeneous charging to be studied independently from $C V$ curve narrowing effects. If experiments are done with RF equipment which quickly measure a $C V$ curve, the effect of the measurement on the device becomes negligible. In this case, $V_{\text {shift }}$ may also be determined by $\left(V_{\mathrm{pi}}^{+}+V_{\mathrm{pi}}^{-}\right) / 2$.

\section{ACKNOWLEDGMENT}

The authors wish to thank J. T. M. van Beek and M. J. E. Ulenaers for fabricating the RF MEMS capacitive switches.

\section{REFERENCES}

[1] G. M. Rebeiz, RF MEMS — Theory, Design, and Technology. New York: Wiley, 2003, ch. 1, p. 1.

[2] E. K. Chan, K. Garikipati, and R. W. Dutton, "Characterization of contact electromechanics through capacitance-voltage measurements and simulations," J. Microelectromechanical Syst., vol. 8, no. 2, pp. 208-217, Jun. 1999.

[3] W. M. van Spengen, R. Puers, R. Mertens, and I. D. Wolf, "A comprehensive model to predict the charging and reliability of capacitive RF MEMS switches," J. Micromech. Microeng., vol. 14, pp. 514-521, 2004.

[4] I. Wibbeler, G. Heifer, and M. Hietschold, "Parasitic charging of dielectric surfaces in capacitive microelectromechanical systems (MEMS)," Sensors Actuators A: Physical, pp. 74-80, Nov. 1998.

[5] X. Yuan, J. C. M. Hwang, D. Forehand, and C. L. Goldsmith, "Modeling and characterization of dielectric-charging effects in RF MEMS capacitive switches," in IEEE MTT-S Int. Microwave Symp. Dig., 2005.

[6] S. Mellé, D. De Conto, L. Mazenq, D. Dubuc, K. Grenier, L. Bary, O. Vendier, J. L. Muraro, J. L. Cazaux, and R. Plana, "Modeling of the dielectric charging kinetic for capacitive RF-MEMS," in IEEE MTT-S Int. Microwave Symp. Dig., 2005.

[7] X. Yuan, S. Cherepko, J. Hwang, C. L. Goldsmith, C. Nordquist, and C. Dyck, "Initial observation and analysis of dielectric-charging effects on RF MEMS capacitive switches," in IEEE MTT-S Int. Microwave Symp. Dig., 2004.

[8] J. R. Reid, R. T. Webster, and L. A. Starman, "Noncontact measurement of charge induced voltage shift in capacitive MEM-switches," IEEE Microwave Wireless Components Lett., vol. 13, Sep. 2003.

[9] H. Nieminen, J. Hyyryläinen, T. Veijola, T. Ryhänen, and V. Ermolov, "Transient capacitance measurement of MEM capacitor," Sensors Actuators A, vol. 117, pp. 267-272, 2005.

[10] X. Rottenberg, B. Nauwelaers, W. D. Raedt, and H. A. C. Tilmans, "Distributed dielectric charging and its impact on RF MEMS devices," in Proc. 34th Eur. Microwave Conf., Amsterdam, The Netherlands, 2004. 


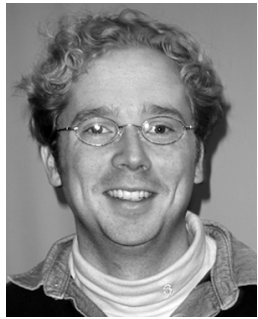

Rodolf W. Herfst received the M.S. degree in applied physics from the Eindhoven University of Technology, Eindhoven, The Netherlands, in 2004. His master thesis was based on research done on laser cooling of iron atoms at the atom optics group of Dr. H. C. W. Beijerinck. He is currently working toward the Ph.D. degree.

$\mathrm{He}$ is working at NXP Semiconductors, Eindhoven, in the device modelling group on the subject of RF MEMS capacitive switches. His research interests include reliability and modelling of RF MEMS capacitive switches.

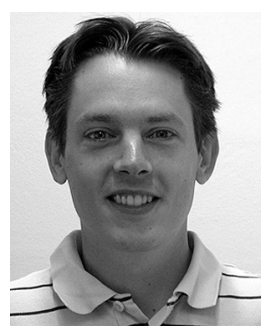

Peter G. Steeneken was born in Groningen, The Netherlands, in 1974. He received the M.Sc. (with honors) and Ph.D. degrees in experimental physics from the University of Groningen, The Netherlands.

From 2002 to 2006, he investigated micro-electromechanical systems at Philips Research. In 2006, he joined NXP Semiconductors, Eindhoven, The Netherlands, and became Project Leader of the RF MEMS Research project. His current interests include the design, simulation, reliability, and characterization of RF MEMS switches.

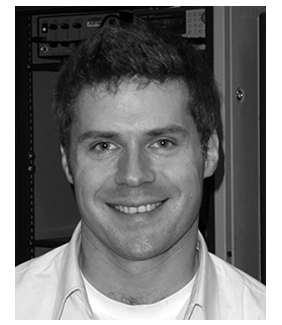

H. G. A. (Bert) Huizing received the M.Sc. and $\mathrm{Ph} . \mathrm{D}$. degrees in electrical engineering, in 1992 and 1996, respectively, from Delft University, The Netherlands, with a focus on solid-state device physics.

In 1996, he joined Philips Research, where he worked and published as Device Physicist on various projects, including fast-switching diodes, high-voltage devices, SiGe bipolar devices, and organic light emitting diodes. In 2003, his interest shifted to building compact models for emerging technologies, such as RF-MEMS switches. Since 2006, he has been a Department Head of a group within NXP Semiconductors, formerly Philips Semiconductors, Eindhoven, The Netherlands, working on device modelling and characterization.

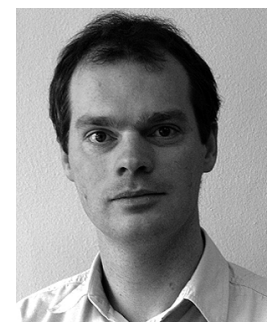

Jurriaan Schmitz (M'02-SM'06) received the M.Sc. degree, in 1990, and Ph.D. degree, in 1994, in experimental physics from the University of Amsterdam, The Netherlands.

$\mathrm{He}$ then joined Philips Research as a Senior Scientist, studying CMOS transistor scaling, characterization, and reliability. Since 2002 , he has been a Full Professor at the University of Twente, Twente, The Netherlands. He has authored or coauthored over 120 journal and conference papers and holds 16 U.S. patents.

Dr. Schmitz has been a TPC member of IEDM, IRPS, ESSDERC, and ICMTS and is a board member of the Dutch Physical Society (NNV). 